\title{
Redefining the Role of Donor Biopsies in the Process of Kidney Graft Assessment
}

\author{
Camillo Carrara $^{a, b}$ Piero Ruggenenti ${ }^{a}{ }^{a}$ Giuseppe Remuzzi ${ }^{a}$ \\ alstituto di Ricerche Farmacologiche Mario Negri IRCCS, Bergamo, Italy; \\ bUnit of Nephrology, Azienda Socio-Sanitaria Territoriale Papa Giovanni XXIII, Bergamo, Italy
}

\section{Keywords \\ Procurement biopsies · Reperfusion biopsies · Kidney transplantation $\cdot$ Marginal donors}

Kidney transplantation is the treatment of choice for patients with end-stage renal disease and is superior to dialysis in terms of long-term mortality risk [1]. Despite the implementation of programs directed to expand criteria for donor selection, there is a steady shortage of kidneys for transplant, and many patients on the waiting list would likely benefit from kidneys that are currently being discarded [2]. In the USA, results of procurement biopsies are the most common reason given for discarding a kidney recovered for transplant [3]. In keeping with this, in recent years, there have been growing skepticism among transplant doctors about the usefulness of donor biopsies [4]. This is not surprising since the criteria to perform, process, and score these biopsies are not standardized. Consequently, studies which have attempt to link histologic findings with subsequent transplant function produced conflicting results [5-7]. Unlike reperfusion biopsies (also called time-zero biopsies), which are paraffin embedded and scored by experienced renal pathologists, procurement biopsies are generally obtained using frozen sections that are often interpreted by a general pathologist without special training in reading kid-

karger@karger.com www.karger.com/nef

(C) 2021 S. Karger AG, Basel

Karger! ney tissue [8]. Morphology of formalin-fixed, paraffinembedded tissue is highly superior to frozen sections. Subtle histology changes are very difficult to assess in the latter. Procurement biopsies are therefore poorly reproducible, do not correlate with scores obtained with paraffin-embedded reperfusion biopsies, and are not significantly associated with transplant outcomes, while reperfusion biopsies provide an excellent prediction of graft survival [9]. These aspects put in question the usefulness of procurement biopsies, at least as how they are currently conceived, in defining the eligibility of kidney grafts for transplantation.

In a recent issue of "Nephron," Perez-Gutierrez et al. [10] provided the results of a single-institution retrospective analysis of 228 deceased donor kidney transplants. The objective of the study was "to describe the correlation of the different histologic characteristics detected by the procurement and reperfusion biopsies in relation to graft function," expressed as glomerular filtration rate at different time points after transplant. Notably, the donors were quite young (median age 47 years), and only 11 of them had a Kidney Donor Profile Index (KDPI) of $85 \%$ or above. Accordingly, most donor biopsies showed no or only mild changes (75.3\%). As expected, given the methodological differences mentioned above, there was great discordance between results obtained from procurement and reperfusion biopsies. For instance, $60.4 \%$ of the grafts 
exhibited more arterial intimal fibrosis and 55.6\% more arteriolar hyalinosis in the reperfusion biopsy than in the procurement biopsy. Overall morphologic details are improved on paraffin than on frozen sections and that might explain why reperfusion biopsies systematically revealed more vascular alterations. One of the main findings of the study was that "vascular alterations in reperfusion biopsies, particularly arterial intimal fibrosis, reliably and persistently correlates with graft function beyond one year after transplant [...]; it is interesting that arterial intimal fibrosis in reperfusion (but not procurement) biopsies is the variable that better correlates with outcome." This led to the following conclusion: "in order to increase the prognostic accuracy of the procurement biopsy, the histology technique should be improved to better evaluate the vasculature."

\section{The Relevance of Standardizing Donor Biopsies' Techniques}

The analysis of Perez-Gutierrez et al. [10] indicates that a precise histologic evaluation of the tissue is crucial for an accurate prediction of graft survival and a correct transplant allocation. One of the issues raised by the authors of the study was that "the 2 types of biopsies were evaluated by different pathologists. While the procurement biopsies were evaluated either by a nephropathologist or a general on-call pathologist, all of the reperfusion biopsies were evaluated by a nephropathologist." In a prospective study, Azancot et al. [11] correlated scores of formalin-fixed, paraffin-embedded donor biopsies provided by one of 12 on-call pathologists with those retrospectively performed by a single renal pathologist blinded to clinical outcomes. The agreement between scores assigned by experienced renal and on-call pathologists was strongly dependent on the training in renal histology of the on-call pathologist. Importantly, only the evaluation of renal biopsies by the renal pathologist was significantly and independently associated with graft function and survival. Moreover, on-call pathologists tended to overcall the amount of chronic changes at the tissue level. On the basis of their readings, more kidneys would have been discarded, and more recipients would have received dual kidney transplantation instead of a single kidney graft [12]: an approach expected to translate into decreased organ utilization and recipient access to available kidneys rather than into optimized organ allocation and increased transplant activity and improved long-term graft outcomes [13]. Thus, pathologist's training and experience greatly impacts the reproducibility of histologic scoring of procurement biopsies, with consequences on organ allocation and outcomes.

Another dilemma concerns the biopsy specimen collection. For procurement biopsies, most centers seem to prefer wedge over needle biopsies because there is the fear of damaging larger blood vessels potentially resulting in uncontrolled bleeding after reperfusion [8]. Perez-Gutierrez et al. [10] observed that: "although wedge biopsies are better suited to collect glomeruli, needle core biopsies sample deeper portions of the cortex and are, therefore, better suited to evaluate the vasculature, which is the strongest and most robust factor related to graft function in our study." All studies comparing wedge with needle biopsies agree that needle biopsies perform much better in the evaluation of vascular lesions because larger branches of interlobular arteries are rarely sampled in wedge biopsies [12, 14].

As mentioned in the introductory paragraph of this commentary, tissue morphology is far better defined on formalin-fixed, paraffin-embedded than on frozen sections [8]. A diagnosis obtained from a frozen section can usually be given $15 \mathrm{~min}$ after the arrival in the pathology lab, while bioptic paraffin-embedded samples takes several hours to be out. However, as demonstrated by PerezGutierrez et al. [10], procurement biopsies using frozen sections often fail in detecting and grading arterial intimal fibrosis, one of the chronic lesions which better correlates with subsequent graft function. Also, even seemingly, quantitative measurements like percentage of glomerulosclerosis are poorly reproducible between procurement and reperfusion biopsies, due to the different procurement and processing method [9]. An additional advantage of paraffin over frozen sections is the use of special stains, such as periodic acid-Schiff and Masson's trichrome, further enhancing morphologic details beyond the limits of traditional hematoxylin and eosin staining [12]. In summary, intrinsic limitations in tissue sampling and processing affect the reliability and prognostic accuracy of reperfusion biopsies and explain the inconsistencies between procurement and reperfusion biopsy findings.

\section{The Matter of Which Donor Graft "Deserves" a Biopsy}

Redefining the role of donor biopsies is not just a question of the operating procedure. The utility of graft histologic assessment for the evaluation of relatively 
young donors with a low KDPI is questionable in particular in limited-resource settings. Instead, this approach may help optimizing the use of organs with high KDPI, which include kidneys from donors with $>60$ years or comorbidities like hypertension or diabetes [15]. These kidneys have a high rate of graft failure, which may result from an imbalance between the number of viable nephrons supplied by older organs and the metabolic demand of the recipient [16]. To fill this gap and improve graft outcomes, transplant of 2 older kidneys in the same recipient has been proposed [17]. The use of standardized histologic criteria to optimize kidney selection from marginal donors and systematically allocate these organs to single or dual transplantation or to discard on the basis of a predefined score of severity of histologic changes was first introduced in 1999 by an international panel of pathologists referring to the Dual Kidney transplant Group (DKG) [18]. Of note, this scoring system, which is based on formalin-fixed, paraffin-embedded sections, was validated in comparative analyses with autoptical samples taken at the same time from the same kidneys [19], and its predictive value was tested in prospective studies of expanded criteria donor kidney transplants $[18,20]$. With this approach, the outcome of histologically evaluated single and dual kidney grafts from marginal or older donors was remarkably superior to that of single grafts from older donors selected according to standard clinical criteria only and similar to that of grafts from young donors. Analogous results were observed when kidneys were procured from donors above the ages of 60 [13,21], 70 [22], or even 80 years [23]. Thus, biopsy-guided allocation of kidneys from older donors may help expanding the donor pool without increasing premature graft loss in everyday clinical practice. Moreover, it has been estimated that an allocation strategy based on a standardized donor biopsy assessment would increase by over $20 \%$ the incidence of renal transplants from donors with high KDPI, which would translate into an overall increase of $4 \%$ considering the entire pool of donors $[24,25]$.

\section{Conclusion}

Given their potential to define organ suitability for transplantation and their role in the choice for dual instead of single kidney transplantation, we believe that procurement biopsies should not be abandoned. However, the study by Perez-Gutierrez et al. [10] brings to our attention the need of stringent methodological rules for a proper scoring of these tissue samples. No doubt, there is urgent need to redefine the way procurement biopsies are currently conceived and performed. First of all, studies aimed at optimizing the methods to collect, process, stain, and score kidney graft samples are welcomed. Second, histologic assessment of donor biopsy should be limited to specific settings, such as organs from older donors or donors with comorbidities that may affect the integrity of kidney tissue such as hypertension, diabetes, and chronic proteinuric nephropathies. Current pitfalls of procurement biopsies should not mislead us regarding the relevance of a precise histologic evaluation to determine organ suitability for transplantation. Critical reassessment and standardization of preimplantation kidney histologic evaluation should be done in the context of an integrated approach, including the combined evaluation of donor and even recipient characteristics to minimize potential mismatches between donor and recipient age, gender, and body weight. Conceivably, avoiding donor/ recipient arterial blood pressure mismatches might help to prevent the risk of posttransplant relative hypoperfusion and impaired functional recovery of kidneys retrieved from hypertensive donors, whose organs are chronically adapted to high perfusion pressures and, then, rapidly exposed to the low perfusion pressures of the postimplantation surgery phase (Carrara et al., in press).

\section{Statement of Ethics}

The authors have no ethical conflicts to disclose.

\section{Conflict of Interest Statement}

The authors have no conflicts of interest to declare.

\section{Author Contributions}

C.C. and P.R. conceived the article contents and prepared the manuscript. G.R. critically revised the final manuscript. All the authors approved the version of the manuscript to be submitted. 


\section{References}

1 Wolfe RA, Ashby VB, Milford EL, Ojo AO, Ettenger RE, Agodoa LY, et al. Comparison of mortality in all patients on dialysis, patients on dialysis awaiting transplantation, and recipients of a first cadaveric transplant. N Engl J Med. 1999;341(23):1725-30.

2 Saidi RF, Hejazii Kenari SK. Challenges of organ shortage for transplantation: solutions and opportunities. Int J Organ Transplant Med. 2014;5(3):87-96.

3 Kasiske BL, Stewart DE, Bista BR, Salkowski $\mathrm{N}$, Snyder JJ, Israni AK, et al. The role of procurement biopsies in acceptance decisions for kidneys retrieved for transplant. Clin J Am Soc Nephrol. 2014;9(3):562-71.

4 Singh P, Farber JL, Doria C, Francos GC, Gulati R, Ramirez CB, et al. Peritransplant kidney biopsies: comparison of pathologic interpretations and practice patterns of organ procurement organizations. Clin Transplant. 2012 May-Jun;26(3):E191-9.

5 Randhawa PS, Minervini MI, Lombardero M, Duquesnoy R, Fung J, Shapiro R, et al. Biopsy of marginal donor kidneys: correlation of histologic findings with graft dysfunction. Transplantation. 2000;69(7):1352-7.

6 Mohan S, Campenot E, Chiles MC, Santoriello D, Bland E, Crew RJ, et al. Association between reperfusion renal allograft biopsy findings and transplant outcomes. J Am Soc Nephrol. 2017;28(10):3109-17.

7 Wang CJ, Wetmore JB, Crary GS, Kasiske BL. The donor kidney biopsy and its implications in predicting graft outcomes: a systematic review. Am J Transplant. 2015;15(7):1903-14.

8 Hopfer H, Kemény É. Assessment of donor biopsies. Curr Opin Organ Transplant. 2013; 18(3):306-12.

9 Carpenter D, Husain SA, Brennan C, Batal I, Hall IE, Santoriello D, et al. Procurement biopsies in the evaluation of deceased donor kidneys. Clin J Am Soc Nephrol. 2018;13(12): 1876-85.
10 Perez-Gutierrez A, Danz D, Chang A, Sekar $\mathrm{P}$, Cummings R, Bachul PJ, et al. Arterial itimal fibrosis in reperfusion biopsy correlates with graft function after kidney transplant. Nephron. 2021;145:150-56.

11 Azancot MA, Moreso F, Salcedo M, Cantarell C, Perello M, Torres IB, et al. The reproducibility and predictive value on outcome of renal biopsies from expanded criteria donors. Kidney Int. 2014;85(5):1161-8.

12 Haas M. Donor kidney biopsies: pathology matters, and so does the pathologist. Kidney Int. 2014;85(5):1016-9.

13 Remuzzi G, Cravedi P, Perna A, Dimitrov BD, Turturro M, Locatelli G, et al. Long-term outcome of renal transplantation from older donors. N Engl J Med. 2006;354(4):343-52.

14 Mazzucco G, Magnani C, Fortunato M, Todesco A, Monga G. The reliability of pretransplant donor renal biopsies (PTDB) in predicting the kidney state. A comparative single-centre study on 154 untransplanted kidneys. Nephrol Dial Transplant. 2010; 25(10):3401-8.

15 Ekser B, Powelson JA, Fridell JA, Goggins WC, Taber TE. Is the kidney donor profile index (KDPI) universal or UNOS-specific? Am J Transplant. 2018;18(4):1031-2.

16 Mackenzie HS, Azuma H, Rennke HG, Tilney NL, Brenner BM. Renal mass as a determinant of late allograft outcome: insights from experimental studies in rats. Kidney Int Suppl. 1995;52:S38-42.

17 Dafoe DC, Alfrey EJ. Dual renal grafts: expansion of the donor pool from an overlooked source. Transpl Int. 1998;11(3):164-8.
18 Remuzzi G, Grinyò J, Ruggenenti P, Beatini M, Cole EH, Milford EL, et al. Early experience with dual kidney transplantation in adults using expanded donor criteria. Double kidney transplant group (DKG). J Am Soc Nephrol. 1999;10(12):2591-8.

19 Randhawa P. Role of donor kidney biopsies in renal transplantation. Transplantation. 2001; 71(10):1361-5.

20 Karpinski J, Lajoie G, Cattran D, Fenton S, Zaltzman J, Cardella C, et al. Outcome of kidney transplantation from high-risk donors is determined by both structure and function. Transplantation. 1999;67(8):1162-7.

21 Pierobon ES, Sefora PE, Sandrini S, Silvio S, De Fazio N, Nicola de F, et al. Optimizing utilization of kidneys from deceased donors over 60 years: five-year outcomes after implementation of a combined clinical and histological allocation algorithm. Transpl Int. 2013;26(8): $833-41$.

22 Rigotti P, Ekser B, Furian L, Baldan N, Valente ML, Boschiero L, et al. Outcome of renal transplantation from very old donors. N Engl J Med. 2009;360(14):1464-5.

23 Ruggenenti P, Silvestre C, Boschiero L, Rota G, Furian L, Perna A, et al. Long-term outcome of renal transplantation from octogenarian donors: a multicenter controlled study. Am J Transplant. 2017;17(12):3159_ 71.

24 Gandolfini I, Buzio C, Zanelli P, Palmisano A, Cremaschi E, Vaglio A, et al. The kidney donor profile index (KDPI) of marginal donors allocated by standardized pretransplant donor biopsy assessment: distribution and association with graft outcomes. Am J Transplant. 2014;14(11):2515-25.

25 Angeletti A, Cravedi P. Making procurement biopsies important again for kidney transplant allocation. Nephron. 2019;142(1):34-9. 